\title{
Determinação da densidade mineral óssea da extremidade distal do rádio-ulna em gatos: correlação entre peso, sexo e idade
}

\author{
Determination the bone mineral density of the distal extremity of the radius-ulna in cats: correlation \\ between weight, sex and age
}

\author{
Luiz Carlos Vulcano ${ }^{\text {I }}$ Flávio Augusto Marques dos Santos ${ }^{\text {II }}$ Carmen Lice Buchmann de Godoy ${ }^{\text {II }}$
}

RESUMO

Os valores de normalidade da densidade mineral óssea (DMO) da extremidade distal de rádio-ulna em 120 gatos clinicamente saudáveis foram determinados usandose a técnica de densitometria óptica em imagens radiográficas. Para a padronização da técnica $e$ interpretação da DMO, foi utilizado um programa computacional especialmente desenvolvido para a medida de densidade óptica em imagens radiográficas, que contém a imagem radiográfica da extremidade distal de rádio-ulna, e uma escala de alumínio (penetrômetro), usada como referencial densitométrico, permitindo a medida da densidade mineral óssea do rádio-ulna correspondente ao valor em milímetros da escala. Os valores médios da densidade mineral óssea da extremidade distal do rádioulna foram de 1,98 $\pm 0,52 \mathrm{mmAl}$ para os machos e de 1,76 $\pm 0,41 \mathrm{mmAl}$ para as fêmeas. Foram observadas correlações estatisticamente significativas entre a DMO, o peso e a idade dos animais estudados $(P<0,0005)$.

Palavras-chave: gatos, rádio-ulna, densidade mineral óssea.

\section{ABSTRACT}

The normal values of bone mineral density (BMD) at the distal end of the radius-ulna were established in 120 clinically healthy cats, using the technique of optical densitometry in radiographic images. The interpretation of BMD was performed using a computer software especially developed for measuring the optical density of the radiographic films, containing radiographic image of the distal extremity of the radius-ulna, and steps of an aluminum scale (penetrometer), used as a densitometric reference. This allowed the measurement of the BMD corresponding to the value in millimeters of the scale. The BMD mean values of the distal end of radius-ulna were: $1.98 \pm 0.52 \mathrm{mmAl}$ for male and $1.76 \pm 0.41$ for female cats. Significant correlations were observed between BMD and weight and BMD and age $(P<0.0005)$.

Key words: cats, radius-ulna, bone mineral density.

\section{INTRODUÇÃO}

A mensuração da densidade mineral óssea (DMO), por meio das mais diversas metodologias, assume atualmente importante papel como nova ferramenta para avaliação das condições nutricionais e de sanidade dos animais. Porém, o alto custo dos equipamentos e, conseqüentemente, dos exames para determinação e avaliação da densidade mineral óssea, inviabilizam a introdução e aplicação da densitometria óssea como modalidade de diagnóstico complementar de rotina, nas condições de trabalho da medicina veterinária brasileira.

Em trabalho de revisão, GRIER et al. (1996) ressaltaram a importância da mensuração do conteúdo mineral ósseo no estudo de doenças metabólicas ósseas, nos processos de consolidação de fraturas, em implantes metálicos e no efeito de fármacos sobre o metabolismo ósseo.

Vários métodos foram desenvolvidos para a avaliação da densidade mineral óssea: a absorção direta por fótons - SPA e DPA(FAULKNER et al., 1991; GENANT et al., 1991), a análise de ativação por nêutrons, a tomografia computadorizada, a fotometria radiográfica (O’CALLAGHAN, 1991), a ultra-sonografia (JEFFCOTT E MCCARTNEY, 1985; MCCARTNEY E

\footnotetext{
IDepartamento de Radiologia e Reprodução Animal, Faculdade de Medicina Veterinária e Zootecnia (FMVZ), Universidade Estadual Paulista (UNESP). Rua Tiradentes, 67, 18611-087, Botucatu, SP, Brasil. E-mail: vulcano@fmvz.unesp.br. Autor para correspondência.

IIPrograma de Pós-graduação em Medicina Veterinária, FMVZ, UNESP, Botucatu, SP, Brasil.

IIIDepartamento de Clínica de Grandes Animais, Universidade Federal de Santa Maria (UFSM), Santa Maria, RS, Brasil.
} 
JEFFCOTT, 1987), a absorciometria radiográfica de dupla energia em animais - DEXA (GRIER et al.,1996) e a densitometria óptica por imagem radiográfica (LOUZADA, 1994).

Por meio dessas técnicas, foi possível correlacionar o conteúdo mineral ósseo com o peso, o sexo e a ocorrência de fraturas em cães de corrida (STOLIKER et al., 1976), avaliar o crescimento de fêmures de cães da raça Beagle e as alterações da atividade óssea em relação à idade destes animais e, ainda, mensurar a densidade mineral óssea de cães da raça Beagle submetidos à intoxicação por chumbo (DELAQUERRIERE-RICHARDSON et al., 1982a, 1982b). Também permitiram determinar a densidade mineral de ossos do tarso e metatarso em cães da raça Greyhound que apresentaram fratura por estresse ou fadiga (EMMERSON et al. 2000) e avaliar a adaptação assimétrica mecânica e densitométrica do quinto osso metacarpiano desses cães (LIPSCOMB et al., 2001).

Apesar de esses estudos mostrarem várias técnicas de quantificação da densidade óssea na medicina veterinária, eles requerem equipamentos de custo elevado, o que inviabiliza a aplicação dessas metodologias como rotina. Embora válidas para avaliação da resistência óssea e do risco de fraturas, as metodologias não-invasivas de mensuração da resistência, estrutura e massa óssea só irão se tornar clinicamente viáveis quando estudos forem realizados visando a facilitar o manuseio do equipamento e a redução de seu custo (FAULKNER et al.,1991).

A densitometria óptica radiográfica (DOR) é um método para mensuração da densidade mineral óssea (DMO) que, por meio da ação fotoquímica da luz sobre emulsões sensíveis, determina a relação existente entre a quantidade de luz recebida em uma película sensível e a quantidade de sal de prata que será reduzida por enegrecimento direto ou por revelador (LOBEL \& DUBOIS, 1973).

Por se tratar de uma técnica recente, a densitometria óptica em imagem radiográfica é ainda pouco empregada na medicina veterinária. Esta metodologia já foi utilizada para avaliar a densidade mineral óssea do carpo ulnar de potros da raça Quarto de Milha, do carpo acessório de potros da raça Puro Sangue Inglês e de eqüinos atletas submetidos a provas de enduro (VULCANO et al., 1997; VULCANO 2001; STERMAN, 2001). Em estudo piloto realizado em 18 cães da raça Rottweiler (9 machos e 9 fêmeas), não foi observada diferença estatisticamente significativa entre a densidade mineral óssea da extremidade distal de rádio dos grupos avaliados (VULCANO et al., 1998). Num outro estudo, no qual se determinou a densidade mineral óssea da extremidade distal de rádio de 293 cães, por meio da técnica de densitometria óptica em imagem radiográfica, observou-se uma correlação positiva entre peso e DMO (LEAL, 2002).

A técnica mostrou-se sensível para avaliar as alterações de densidade mineral óssea em felinos frente ao tipo de manejo alimentar a que os grupos de estudos eram submetidos (GALLO et al., 1996) e foi mais eficiente do que as análises bioquímicas séricas de fosfatase alcalina, fósforo e cálcio para a determinação da desminerilização óssea em felinos, provocada por hiperparatioreodismo nutricional secundário induzido (RAHAL et al., 2002).

Visando a padronizar os valores referenciais normais da densidade mineral óssea nos felinos domésticos, este estudo teve por objetivo determinar os valores da DMO da extremidade distal do rádioulna por meio da técnica de densitometria óptica em imagem radiográfica, correlacionando os valores encontrados com o peso, o sexo e a idade.

\section{MATERIAL E MÉTODOS}

Foram selecionados felinos domésticos clinicamente sadios de raças variadas a partir dos três meses de idade, encaminhados ao setor de Radiologia do Hospital Veterinário da FMVZ, UNESP, Campus de Botucatu. Todos os animais com idade conhecida e confirmada pelos proprietários foram pesados e divididos em grupos por idade (fechamento epifisário), sexo e raça.

As radiografias simples foram realizadas no membro anterior direito, região do rádio-ulna, em projeção crânio-caudal, com os animais em decúbito esternal, utilizando-se aparelho radiográfico convencionala ${ }^{a}$ com distância foco-filme de $90 \mathrm{~cm}$, chassis metálicos $18 \times 24 \mathrm{~cm}$, com telas intensificadoras ${ }^{\mathrm{b}}$ e filmes radiográficos ${ }^{\mathrm{c}} 18 \times 24 \mathrm{~cm}$. O chassi foi acondicionado dentro do porta-chassi, onde estava afixado o penetrômetro de alumínio de liga específica ${ }^{\mathrm{d}}$, usado como referencial densitométrico (Figura 1). A quilovoltagem $(\mathrm{kVp})$ padronizada foi de $40 \mathrm{kVp}$, e a miliamperagem por segundo ( $\mathrm{mAs}$ ) foi determinada de acordo com o porte do animal, sendo 2,5mAs o valor mínimo e 4,5mAs o valor máximo. Os filmes radiográficos foram revelados em processadora automática padrão ${ }^{\mathrm{e}}$.

As radiografias foram digitalizadas por meio de scanner de mesa $\mathrm{HP}^{\mathrm{f}}$, modelo scanjet 6C, com adaptador para transparência $\mathrm{HP}^{\mathrm{g}}$, modelo scanjet 6C/ T, utilizando-se o programa computacional Deskscan. Posteriormente, as imagens foram recapturadas e analisadas pelo programa computacional ${ }^{\mathrm{h}}$ especialmente desenvolvido para este projeto (Figura 2). Através da 


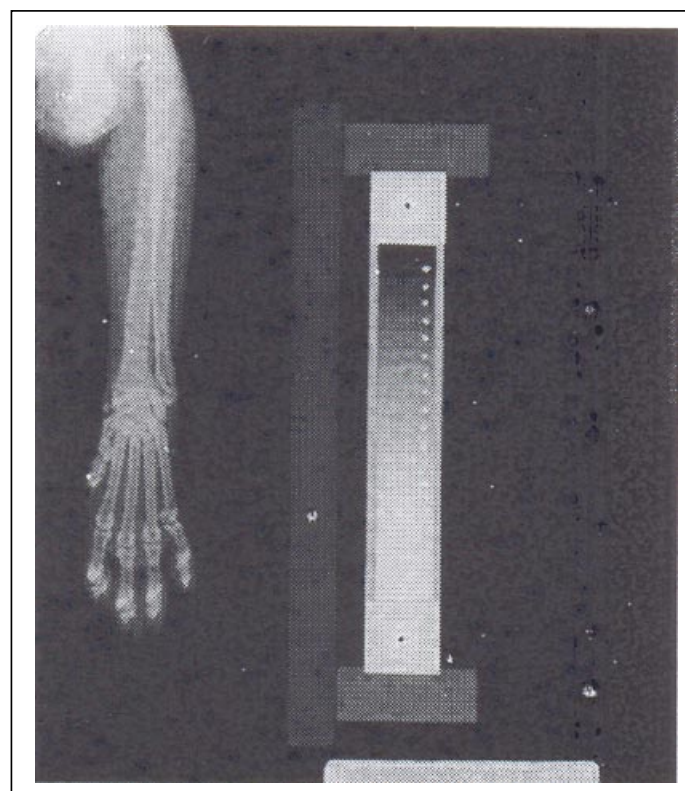

Figura 1 - Radiografia de rádio-ulna de gato, projeção crânio-caudal, junto à escala de alumínio.

comparação entre densidade média da região distal de rádio-ulna selecionada com a densidade média do penetrômetro, determinou-se a densidade mineral óssea, com valores expressos em milímetros de alumínio (mmAl).

Para a relação da idade, do peso e da densitometria óssea, em função de a raça, sexo e fechamento do disco epifisário, utilizou-se a estatística não-paramétrica de Mann-Withney, cujos resultados foram apresentados em tabelas contendo medidas descritivas de posição e variabilidade (NORMAN \& STREINER, 1994).

Para o estudo da associação da densitometria óssea com idade e peso, considerando a

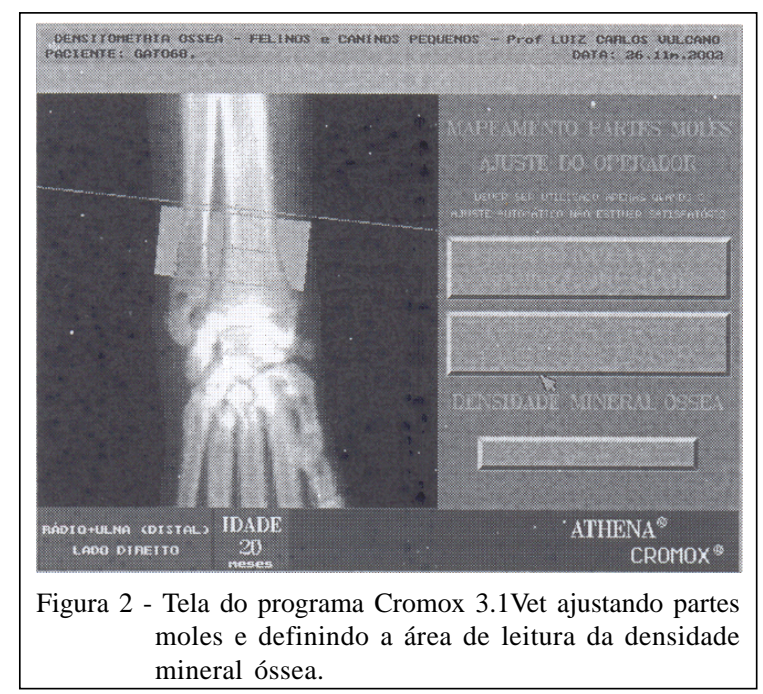

população geral e classificada por raça, sexo e fechamento do disco epifisário, utilizou-se o coeficiente de correlação de Spearman (NORMAN \& STREINER, 1994). Todas as discussões dos resultados foram realizadas no nível de 5\% de significância.

\section{RESULTADOS E DISCUSSÃO}

Foram radiografados 120 animais, sendo que, deste total, 104 gatos (86,67\%) não possuíam raça definida (SRD) e $16(13,33 \%)$ eram da raça siamesa; sendo 66 machos (55\%) e 54 fêmeas (45\%). Quarenta e cinco $(37,5 \%)$ animais apresentavam disco epifisário aberto, enquanto que 75 (62,5\%) tinham o completo fechamento epifisário.

As medidas descritivas mostraram que a idade média da população avaliada foi de 27,6 meses, com mediana de 17 meses. O peso corpóreo médio foi de $3,2 \pm 1,1 \mathrm{~kg}$ e a densidade mineral óssea média da população estudada foi de $1,88 \pm 0,48 \mathrm{mmAl}$, com mediana de $1,80 \mathrm{mmAl}$.

Os machos apresentaram densidade mineral óssea média de 1,98 $\pm 0,52 \mathrm{mmAl}$, e as fêmeas de 1,76 \pm $0,41 \mathrm{mmAl}$. Os gatos que tinham o disco epifisário aberto apresentarem densidade mineral óssea média de $1,73 \pm 0,38 \mathrm{mmAl}$, enquanto que os animais de epífise fechada apresentaram DMO de 1,97 $\pm 0,51 \mathrm{mmAl}$. A medida da associação da densidade mineral óssea (DMO) com idade e peso, no geral e por raça e segundo sexo e fechamento epifisário, estão apresentadas nas tabelas 1 e 2, respectivamente (correlação de Spearman). Foram observadas correlações estatisticamente significativas entre a densidade mineral óssea (DMO) e o peso e a idade nos animais estudados $(\mathrm{P}<0,0005)$.

$\mathrm{Na}$ comparação entre os sexos, houve diferença estatisticamente significativa entre as médias dos valores de densidade mineral óssea de machos e fêmeas $(\mathrm{P}<0,05)$. $\mathrm{O}$ mesmo resultado foi obtido por STOLIKER et al. (1976), que relataram a correlação entre os valores de densidade mineral óssea e o sexo, embora a metodologia e a amostragem sejam diferentes às deste estudo.

O estudo mostrou uma forte associação entre os valores da densidade mineral óssea e o peso corpóreo da população estudada $(\mathrm{P}<0,0005)$, ou seja, animais mais pesados apresentam uma maior densidade

Tabela 1 - Medida da associação da densidade mineral óssea (DMO) com idade e peso, no geral e por raça (correlação de Spearman).

\begin{tabular}{lclc}
\hline Associação & Geral $(120)$ & \multicolumn{1}{c}{ SRD $(104)$} & Siamês (16) \\
\hline Idade x DMO & $0,32(\mathrm{P}<0,0005)$ & $0,30(\mathrm{P}<0,005)$ & $0,33(\mathrm{P}>0,05)$ \\
Peso x DMO & $0,37(\mathrm{P}<0,0005)$ & $0,38(\mathrm{P}<0,0005)$ & $0,43(\mathrm{P}>0,05)$ \\
\hline
\end{tabular}


Tabela 2 - Medida da associação da densidade mineral óssea (DMO) com idade e peso, segundo sexo e fechamento epifisário (correlação de Spearman).

\begin{tabular}{lllll}
\hline & & Sexo & & Fechamento \\
\cline { 2 - 5 } Associação & Macho(66) & Fêmea(54) & Aberto(45) & Fechado(75) \\
\hline Idade x DMO & $0,29(\mathrm{P}<0,05)$ & $0,40(\mathrm{P}<0,005)$ & $0,24(\mathrm{P}>0,05)$ & $0,29(\mathrm{P}<0,05)$ \\
Peso x DMO & $0,28(\mathrm{P}<0,05)$ & $0,41(\mathrm{P}<0,005)$ & $0,32(\mathrm{P}<0,05)$ & $0,31(\mathrm{P}<0,01)$ \\
\hline
\end{tabular}

mineral óssea. Esta correlação foi observada por STOLIKER et al. (1976), DELAQUERRIERERICHARDSON et al. (1982a), MARTIN et al. (1981) e LEAL (2002). Isto, segundo LIPSCOMB et al. (2001), pode ser explicado pelo fato de o esqueleto ser uma estrutura peso-dependente, adaptando-se ao peso a ele conferido por meio de osteogênese e aumento de massa óssea.

$\mathrm{Na}$ comparação entre os animais que apresentavam disco epifisário aberto e fechado, houve diferença estatisticamente significativa entre os grupos para as médias dos valores de DMO obtidas $(\mathrm{P}<0,05)$.

$\mathrm{O}$ estudo mostrou uma associação significativa entre os valores da densidade mineral óssea e a idade da população estudada $(\mathrm{P}<0,0005)$. Deste modo, animais mais velhos apresentam uma densidade mineral óssea maior, divergindo daqueles obtidos por DELAQUERRIERE-RICHARDSON et al. (1982a), VULCANO et al. (1998) e LEAL (2002).

$\mathrm{Na}$ associação da densidade mineral óssea com idade, segundo o sexo, houve correlação positiva para sexo, sendo significativa para a população de fêmeas $(\mathrm{P}<0,005)$. Este dado confronta-se com os obtidos por LEAL (2002), que encontrou uma correlação negativa entre os valores de DMO e a idade da população de fêmeas caninas. Contudo, pelo fato de a média de idade da população canina estudada ser maior do que a observada no presente estudo e em outra espécie, trabalhos futuros poderiam estabelecer uma melhor correlação entre idade, sexo e densidade mineral óssea de gatos.

\section{CONCLUSÕES}

O estudo, nas condições em que foi realizado, permitiu concluir que os valores médios da densidade mineral óssea (DMO) da extremidade distal do rádio-ulna para os animais adultos machos são de $1,98 \pm 0,52 \mathrm{mmAl}$ e, para as fêmeas, de $1,76 \pm 0,41 \mathrm{~mm}$ Al. Para animais com o disco epifisário aberto na extremidade distal do rádio-ulna, os valores médios da DMO são de $1,73 \pm 0,38 \mathrm{~mm} \mathrm{Al}$, enquanto que, para aqueles com disco epifisário fechado, são de 1,97 \pm 0,51mm Al.
Animais mais pesados e mais idosos têm valor de DMO proporcionalmente maior.

\section{AGRADECIMENTOS}

À Fundação de Amparo à Pesquisa do Estado de São Paulo (FAPESP), pelo apoio concedido.

\section{FONTES DE AQUISIÇÃO}

aTUR 800, $500 \mathrm{~mA}$

${ }^{\mathrm{b}}$ Kodak Lanex X-Omatic Regular Screens (Kodak Eastman Company).

${ }^{\mathrm{C} B r a f}$ - Kodak Brasileira Com e Ind. Ltda.

${ }^{\mathrm{d}}$ Liga 6063 - ABNT (Brasil).

${ }^{\mathrm{e}}$ Macrotec - Ind. e Com. De Equipamentos Ltda.

${ }^{\mathrm{f}}$ Hewlett Packard.

${ }^{8}$ Hewlett Packard.

hAthena - SAI - Sistema de Inteligências Avançada Com. Imp. Exp. Ltda. - SJC - SP

\section{REFERÊNCIAS}

DELAQUERRIERE-RICHARDSON, L. et al. Radiographic morphometry and radiographic photodensitometry of the femur in the Beagle at 13 and 21 months. American Journal Veterinary Research, v.43, n.12, p.2255-2258, 1982a.

DELAQUERRIERE-RICHARDSON, L. et al. Radiographic studies on bone in Beagles subjected to low levels of dietary since birth. Veterinary and Human Toxicology, v.24, n.6, p.401-405, $1982 b$.

EMMERSON, T.D., et al. Dual-energy X-ray absorptiometry measurement of bone-mineral density in the distal aspect of the limbs in racing Greyhounds. American Journal Veterinary Research, v.61, n.10, p.1214-1219, 2000.

FAULKNER, K.G. et al. Noninvasive measurements of bone mass, structure, and strength: current methods and experimental techniques. American Journal Roentgenology, v.157, p.1229-1237, 1991.

GALLO, R.N. et al. Avaliação da densidade óssea em gatos em crescimento submetidos a dois tipos de ração. In: CONGRESSO PANAMERICANO DE CIÊNCIAS VETERINÁRIAS, 15., 1996, Campo Grande. Anais... Mato Grosso do Sul: PANVET, 1996. p.78.

GENANT, H.K. et al. Measurement of bone mineral density: current status. America Journal Medicine, v.51, suppl. 5B, p.49S-53S, 1991. 
GRIER, S.J. et al. The use of dual energy x-ray absorptiometry in animals. Investigative Radiology, v.31, n.1, p.50-62, 1996.

JEFFCOTT, L.B.; McCARTNEY, R.N. Ultrasound as a tool for assessment of bone quality in the horse. Veterinary Record, v.116, p.337-342, 1985.

LEAL, A.C.R. Determinação dos valores normais da densidade mineral óssea (DMO) da extremidade distal do rádio em cães por meio da técnica de densitometria óptica radiográfica em imagens radiográficas: correlação entre o peso, sexo e idade. 2002. 51f. Dissertação (Mestrado em Cirurgia Veterinária) - Curso Pós-graduação em Medicina Veterinária, Faculdade de Medicina Veterinária e Zootecnia Universidade Estadual Paulista, Campus de Botucatu.

LIPSCOMB, V.J. et al. Asymmetric densitometric and mechanical adaptation of the left fifth metacarpal bone in racing greyhounds. Veterinary Record, v.148, p.308-311, 2001.

LOBEL, L.; DUBOIS, M. Manual de sensiometria óptica: la técnica de la medición de los materiales fotográficos. 2.ed. Barcelona: Ediciones Omega, 1973. 238p.

LOUZADA, M.J.Q. Otimização da técnica de densitometria óptica em imagens radiográficas de peças ósseas. Estudo In vivo. 1994. 191f. Tese (Doutorado Engenheira Biomédica) - Faculdade de Engenharia Elétrica, Universidade Estadual de Campinas, UNICAMP.

MARTIN, R. et al. Bone loss in the Beagle tibia: influence of age, weight and sex. In: JEE, W.S.S.; PARFITT, A.M. Bone histomorphometry. Paris: SNPMD, 1981. 497p.

McCARTNEY, R.N., JEFFCOTT, L.B. Combined $2.25 \mathrm{MHz}$ ultrasound velocity and bone mineral density measurements in the equine metacarpus and their in vivo applications. Medical \& Biological Engineering \& Computing, v.25, p.620-626, 1987.
NORMAN, G.R.; STREINER, D.L. Biostatistics - The base essential. St. Louis: Mosby-YearBook, 1994. 260p.

O’CALLANGHAN, M.W. A brief look at new technologies and their potencial application to equine diagnosis. Veterinary Clinics of North America Equine Practice, v.7, n.2, p.467469, 1991.

RAHAL, S.C. et al. Densitometría óptica rdiográfica na avaliação do hiperparatireoidismo secundário nutricional induzido em gatos jovens. Ciência Rural, v.32, n.3, p.421425, 2002

STERMAN, F.A. Avaliação da densidade mineral óssea em eqüinos atletas destinados ao enduro eqüestre pelo método de densitometria óptica radiográfica. 2001. 47f. Tese (Livre-Docência) - Faculdade de Medicina Veterinária e Zootecnia, Universidade de São Paulo.

STOLIKER, H.E. et al. Bone mineral measurement by photon densitometry in racing sled dogs, and its relationship to body weight, sex and bone fractures. Veterinary Medicine Small Animal Clinician, v.11, p.1545-1550, 1976.

VULCANO, L.C. et al. Carpo ulnar bone density in quarter horse foals measured by radiographic optical densitometry. Hora Veterinária, v.100, p.52-54, 1997.

VULCANO, L.C. et al. Determination of the normal values of density of the radius in Rotweilers, using radiographic otical densitometry (experimental study), Buenos Aires, Argentina, 1998. In: CONGRESS OF THE WORDL SMALL ANIMAL VETERINARY ASSOCIATION, 23., 1998 Buenos Aires. Proccedings... Argentina: WSAVA, 1998. p.767

VULCANO, L.C, Determinação e padronização dos valores normais da densidade mineral óssea (DMO) do carpo acessório de eqüinos em crescimento, da raça Puro Sangue Inglês (PSI), por meio da densitometria óptica em imagem radiográfica. 2001. 62f. Tese (Livre-Docência) - Faculdade de Medicina Veterinária e Zootecnia, Universidade Estadual Paulista, Campus de Botucatu. 